Pneumologe 2022 · 19:61-62

https://doi.org/10.1007/s10405-022-00439-z Angenommen: 2. Februar 2022

(C) The Author(s), under exclusive licence to Springer Medizin Verlag GmbH, ein Teil von Springer Nature 2022

Redaktion

S. Kluge, Hamburg

\section{Entwicklung und Fortschritt}

\author{
Stefan Kluge \\ Klinik für Intensivmedizin, Universitätsklinikum Hamburg-Eppendorf, Hamburg, Deutschland
}

Liebe Kolleginnen und Kollegen,

in der Hand halten Sie die DGP-Kongressausgabe zum 62. Kongress der Deutschen Gesellschaft für Pneumologie und Beatmungsmedizin.

Die Corona-Pandemie war in den letzten 2 Jahren omnipräsent, und wir haben uns im medizinischen und pneumologischen Bereich intensiv damit befasst. Aber auch in anderen Bereichen der Pneumologie hat es in den letzten Jahren wesentliche Fortschritte gegeben.

Thematisiert werden in diesem Themenheft daher analog zum Kongressmotto „Entwicklung und Fortschritt" die Innovationen bei zentralen Themen der Pneumologie.

Die Pandemie und die Ausbreitung multiresistenter Erreger zeigen eindrucksvoll, dass trotz aller medizinischer und technischer Fortschritte Infektionskrankheiten weiterhin eine globale Bedrohung darstellen. Mathias Pletz geht in seinem Beitrag auf Fortschritte bei den Themen Erregerdiagnostik, neue Antiinfektiva und Vakzine ein. Er streift dabei zum Schluss auch das Thema COVID-19.

\section{》) In der Pneumologie hat es in den letzten Jahren wesentliche Fortschritte gegeben}

Christian Taube beleuchtet die Entwicklungen und Fortschritte bei der Diagnostik und Therapie von obstruktiven Atemwegserkrankungen. Er zeigt auf, dass es sowohl bei Patienten/innen mit Asthma als auch bei Patienten/innen mit COPD notwendig ist, die Entzündungsreaktion zu erfassen.
So ist bei Patienten/innen mit Erhöhung der inflammatorischen Parameter die Behandlung oder ggf. Intensivierung der antientzündlichen Behandlung notwendig.

Das dem Verfasser dieser Zeilen sehr naheliegende Thema Beatmungsmedizin wird von Wolfram Windisch und Bernd Schönhofer dargestellt. Dabei geht es um die akute Anwendung in der Intensivmedizin, die Respiratorentwöhnung (Weaning) und die Langzeitanwendung und damit die außerklinische Beatmung. Vor allem beim Thema der außerklinischen Beatmung setzen die beiden Autoren kritische Akzente und weisen auf die hohe Zunahme an Patienten/innen mit außerklinischer Beatmung in Deutschland hin und thematisieren Fehlanreize.

Die häufigste solide onkologische Organerkrankung mit der schlechtesten Prognose ist das Lungenkarzinom. Christian Grohé weist in seinem Beitrag auf die dynamischen Entwicklungen im Bereich des Lungenkarzinoms in Bezug auf Diagnostik und Therapie hin. Übersichtlich stellt er Neuerungen bei der medikamentösen Therapie dar, er weist aber auch darauf hin, dass die Therapie von Patienten/innen mit einem Lungenkarzinom heutzutage eine multidisziplinäre interprofessionelle Aufgabe ist.

Auch im Bereich der interstitiellen Lungenerkrankungen (ILD) waren in den letzten Jahren sehr große Fortschritte in Diagnostik und Therapie zu verzeichnen. Zum Abschluss geben Katharina Buschulte et al. daher zu diesem Thema einen Überblick, der viele wichtige Aspekte wie u.a. Biomarker, bildgebende Verfahren 


\section{Einführung zum Thema}

sowie neue nicht- und medikamentöse Therapieverfahren beinhaltet.

Ich möchte mich bei den Autoren/ innen für die hervorragenden Beiträge bedanken und wünsche Ihnen eine interessante Lektüre!

Prof. Dr. Stefan Kluge

Kongresspräsident DGP-Kongress 2022

\section{Korrespondenzadresse}

Prof. Dr. med. Stefan Kluge

Klinik für Intensivmedizin, Universitätsklinikum Hamburg-Eppendorf

Martinistr. 52, Gebäude Ost 10, 20246 Hamburg, Deutschland

skluge@uke.de

Interessenkonflikt. S. Kluge gibt an, dass kein Interessenkonflikt besteht.

\section{MED UPDATE SEMINARE 2022}

Pneumo Update 2022

19. Pneumologie-Update-Seminar

11.-12. November 2022

Mainz und Livestream

18.-19. November 2022

Berlin und Livestream

\section{Wiss. Leitung:}

Prof. Dr. Felix Herth, Heidelberg

Prof. Dr. Michael Pfeifer, Donaustauf Prof. Dr. Claus Vogelmeier, Marburg

Prof. Dr. Tobias Welte, Hannover

Unter der Schirmherrschaft der DGP, DGIM

www.pneumo-update.com
Allergo Update 2022

12. Allergologie-Update-Seminar

18.-19. März 2022

Berlin und Livestream

\section{Wiss. Leitung:}

Prof. Dr. Jörg Kleine-Tebbe, Berlin Prof. Dr. Christiane Bayerl, Wiesbaden Prof. Dr. Kirsten Beyer, Berlin Prof. Dr. Martin Wagenmann, Düsseldorf Prof. Dr. Thomas Werfel, Hannover

Unter der Schirmherrschaft der DGAKI, DGIM

www.allergo-update.com

Auskunft für alle Update-Seminare: med update $\mathrm{GmbH}$ www.med-update.com

Tel.: 0611 - 736580

info@med-update.com 